\title{
A novel fabrication set-up for the flexible production of silicone based EAP "artificial muscle" actuators
}

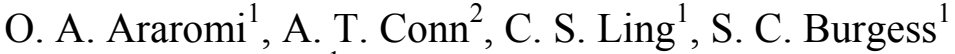 \\ \& R. Vaidyanathan ${ }^{1}$ \\ ${ }^{I}$ Department of Mechanical Engineering, University of Bristol, UK \\ ${ }^{2}$ Department of Engineering Mathematics, University of Bristol, UK
}

\begin{abstract}
This paper presents a novel fabrication set-up for producing silicone based EAP "artificial muscle" actuators which is relatively simple, flexible and well suited to low volume production. The required apparatus can be constructed quickly and at a low cost compared to competing techniques. The set-up can produce multi-layered configurations for applications that require greater actuator strain or stress. The set-up has also been demonstrated to produce individual polymer layers less than $100 \mu \mathrm{m}$ thick, which is beneficial as for a given input voltage actuator performance improves with decreasing layer thickness. A case study is presented to demonstrate the quality of the set-up and a potential bio-inspired application of the fabricated actuator is outlined.
\end{abstract}

Keywords: electroactive polymer, dielectric elastomer, micro air vehicle.

\section{Introduction}

Electroactive polymers (EAPs) are high strain actuators based on the deformation of polymeric materials under electrical stimulation. EAPs are a rapidly developing technology and are attracting interest because of their inherent compliance, light weight properties, simple actuation mechanisms and large energy densities. Moreover, unlike other traditional actuation technologies, such as pneumatic or hydraulic actuators, EAP actuators do not require large or bulky peripheral devices, such as pumps or compressors. Dielectric elastomer actuator (DEA) type EAPs in particular have been shown to have very large 
actuation strains (up to $300 \%$ with acrylic VHB 4910) and this means that there is less need for mechanical amplification in many applications [1], which can often reduce efficiency. EAPs are often referred to as 'artificial muscles' as they have similar performance characteristics to natural muscles (Figure 1). As such, EAPs have been widely investigated in the field of bio-inspired robotics [2-5]. Of particular interest are the series of biomimetic walking robots developed by Pei et al. [4] at SRI International which use DEAs as 'leg' structures to provide insect-like locomotion.

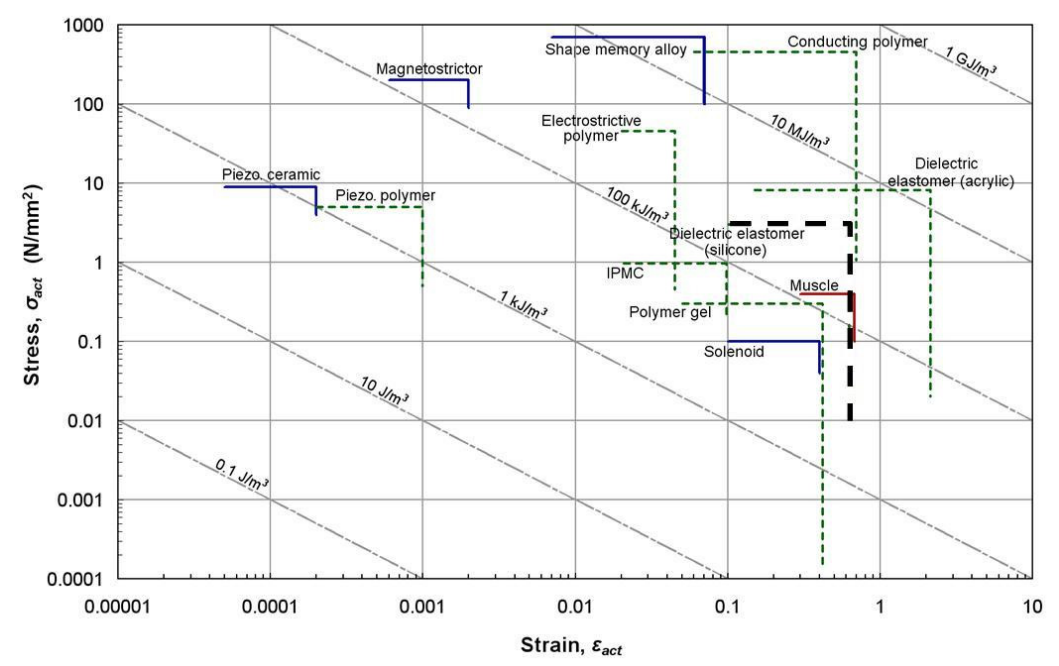

Figure 1: Maximum stress-strain performance limits for conventional actuators (solid lines), biological muscle (red line) and electroactive polymer actuators (black dashed line) (from [3]).

DEAs are made up of an elastomer film sandwiched between two compliant electrodes, essentially forming an 'elastic capacitor'. When a large voltage field (typically around 50 to $200 \mathrm{MV} / \mathrm{mm}$ [1]) is applied across the electrodes, coulomb forces cause compression of the film in the thickness direction and expansion of the film in planar direction (Figure 2). The effective induced pressure in the thickness direction is often referred to as the Maxwell stress, $p$, given by (1).

$$
p=e_{r} e_{0}\left(\frac{V}{h}\right)^{2}
$$

where $e_{r}$ is the relative dielectric constant of the elastomer film, $e_{0}$ is the permittivity of free space (equal to $8.85 \times 10^{-12} \mathrm{~F} / \mathrm{m}$ ), $h$ is the initial film thickness and $V$ is the voltage across the elastomer film.

The manufacture of functional dielectric elastomer actuators (DEAs) can be non-trivial. Current fabrication methods are often intricate and highly optimised for a specific actuator configuration or for a specific dielectric material. A 


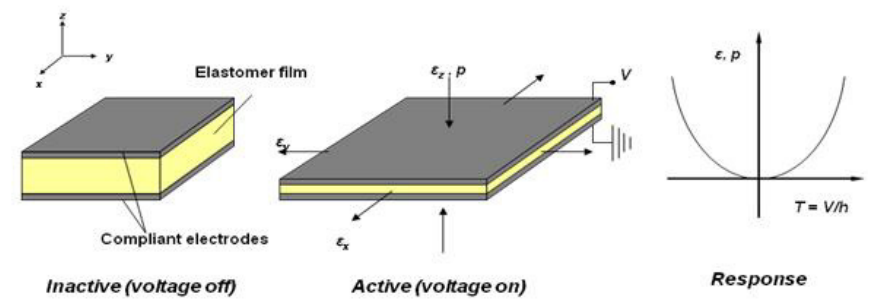

Figure 2: $\quad$ DE actuation principle.

functional DEA can require several dielectric layers which can further complicate the fabrication process, especially if stacking is performed manually [6]. In addition, it is very important to have consistency and accuracy in the thickness of layers which can be difficult to maintain at small thicknesses (e.g. < $100 \mu \mathrm{m}$ ) without the use of expensive equipment. Non-uniform layers may inhibit the performance of a DEA and increase the risk of actuator failure. Thin layers are more likely to 'breakdown' electrically, which would result in the actuator short-circuiting. Quality of electrical connections between alternate layers is another important consideration, especially for large multilayer configurations.

Many research laboratories are producing novel configurations or designs which do not have establish fabrication processes associated with them. In addition, growth of interest in the DEA research field means that many research institutions have limited knowledge in the fabrication of DEAs and may require may a quick and low-cost method for fabricating functional DEAs.

The key requirements for an ideal DEA fabrication system can be summarised as follows:-

- The ability to produce very thin $(<100 \mu \mathrm{m})$ elastomer film layers.

- The ability to apply thin compliant electrodes with good bonding to the elastomer film whilst maintaining good electrical conductivity.

- The ability to produce multiple layer DEAs.

- The ability to provide mechanically sound bonding between layers in multilayer configurations for improved performance and structural integrity.

- Consistent and accurate layer thicknesses.

- Flexibility to vary shape or dimensions of the actuator.

- Low fabrication time.

- Good repeatability and reliability.

- Free from impurities that could create non-uniformities in the silicone layers or a short-circuit path.

The ability to produce very thin layers is important because the actuation stress (and hence actuation strain), $p$, is inversely proportional to the square of the thickness, $h$, of the layers for a given voltage as eqn (1) shows. Thus, in general it is better to have a large number of thinner elastomer layers than to have a smaller number of thicker layers. Also, the speed of response of a DEA to a driving signal can be improved by having an increased number of dielectric 
layers. This is due to the fact that the time taken to apply a desired potential across an electrode pair is proportional to the resistance and capacitance of the electrodes (commonly known as the $R C$ time constant). Since for a given electrode material the surface area of the electrodes determines the $R C$ time constant, splitting up the total electrode surface area into discrete elements with individual electrical connections will reduce the effective area and increase speed of response. Of course, adding numerous electrical connections to one large electrode would have the same effect as segmenting the electrode area, but this is typically impractical since the electrodes must be highly compliant.

With multilayer structures, interlayer bonding can be an important issue for the structural integrity of the actuator, especially if the actuator is required to hold a load in both its passive and active states [7]. Interlayer bonding is also important for preventing arcing of charge from around the side of a layer and also for electrode adhesion which is particularly important if the silicone elastomer being used is not naturally adhesive [7].

Flexibility in the geometry and shapes of the actuators produced is essential when developing prototype actuators. Silicone elastomers are easy to work with, inexpensive and can be fabricated in thin films without prestraining, which is necessary for other dielectric materials such as acrylic VHB 4910 (3M). This prestraining can reduce the power density of the actuators produced if done mechanically, or require complex and expensive equipment if done using other methods [8]. Hence, silicone elastomers will be the only dielectric material type utilised in this work.

Specialist methods for fabricating DEAs have been developed by various research groups and institutions [9-11], all of which are effective in producing high quality actuators. Polypower is a commercial company which supply preelectroded silicone based dielectric elastomer films with a film thickness of $70 \mu \mathrm{m}$ and high conductivity electrodes. However, their fabrication process is complex, intricate and optimised for large volume production of single film actuators. Their equipment is also large, expensive and therefore non ideal for small laboratories. The fabrication process outlined in $[9,10]$ is the most similar to the work presented here, however the spin coating equipment used is expensive, requires accurate speed and acceleration control and is limited in its flexibility for producing different shapes and dimensions.

This paper presents a novel flexible fabrication set-up for producing dielectric elastomer EAP actuators which is relatively simple and accurate and well suited to low volume production.

\section{Wheel spraying method}

Figure 3 summarises the proposed DE fabrication set up and method developed at the University of Bristol:

\subsection{Preparation}

Dow Corning Silastic 3481 silicone base (Part A) and curing agent (Part B) are first mixed together thoroughly according to their mixture ratio then dissolved in 


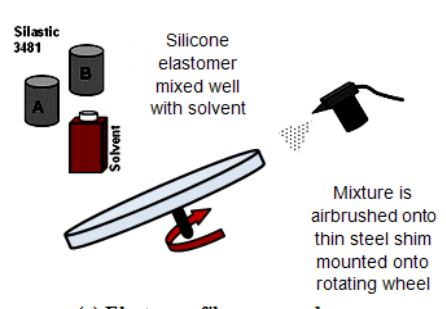

(a) Elastomer film prepared

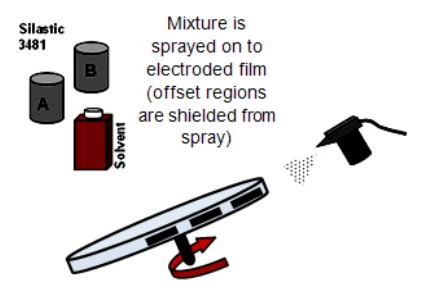

(c) Additional layers sprayed directly on top of previous layers

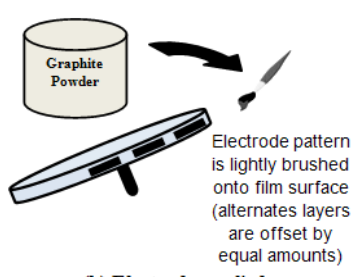

(b) Electrode applied

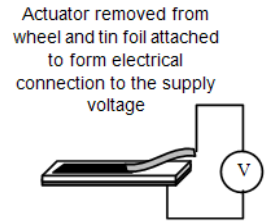

(d) Actuator electrical terminals fabricated

Figure 3: Fabrication process of dielectric elastomer multilayer DEA.

a solvent to form a relatively low viscosity liquid adequate for airbrushing. The solvent-silicone mixture is poured into the airbrush container through a strainer to remove any undissolved elastomer clumps. The mixture is then airbrushed onto the circumference of a rotating wheel which has steel shim sections mounted to it. The dimension of the shim sections depend on the shape of the DEA being fabricated. The circumference of the wheel is lined with magnetic tape to ensure the shim stays flush with the wheel circumference during spraying. The wheel is attached to a DC motor spinning at constant velocity to ensure a constant layer thickness. The airbrush tip is angled such that the spray flow falls on the centre line of the shim sections.

\subsection{Electrode application}

After the spraying process is complete, the solvent is allowed to evaporate leaving the silicone layer behind. Once this layer has fully cured, graphite powder electrodes are lightly brushed onto the surface of the silicone through a mask. The masks are made from acetate transparency films coated with a thin layer of tacky silicone (BJB TC-5005) underside to provide good adhesion of the mask to the film layer whilst the electrode is being applied. This also allows the masks to be re-used. A $5 \mathrm{~mm}$ offset is added to the electrode mask pattern to allow alternate electrode layers to be connected in parallel (Figure 4). Datum marks are also drawn onto the mask to ensure the electrodes line up through the layers. 


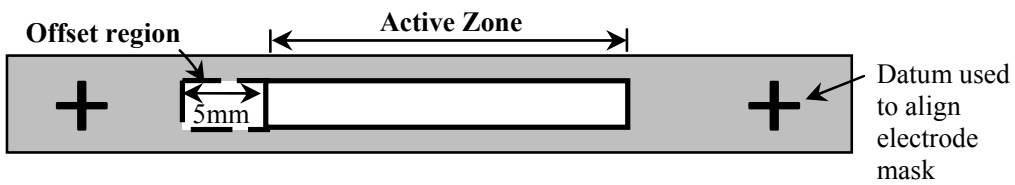

Figure 4: Electrode mask pattern.

\subsection{Additional layers}

The next elastomer layer can be immediately airbrushed directly on top of the previously electroded film. The offset region is covered with tape to shield it from the spray flow. This is to allow latter electrode layers to be electrically connected through the actuator. Different charge polarities need to be applied to electrodes on alternate layers for the stack configuration to function, i.e. the electrodes must be connected electrically in parallel. To accomplish this, the electrode is staggered by the offset distance (i.e. $5 \mathrm{~mm}$ ) to allow the actuator to be connected in parallel as shown in Figure 5. Finally, a thin final layer of silicone is airbrushed on top of the last electrode for electrical insulation.

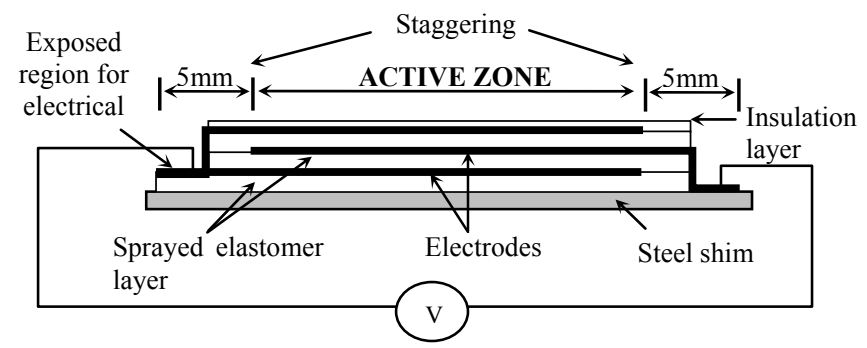

Figure 5: Schematic of the sprayed multilayer structure with electrode connections.

\subsection{Supply voltage terminals}

The shim is removed from the wheel and strips of aluminium foil are attached to the offset regions to provide the electrical connection for the input driving voltage. Depending on the desired actuator configuration, the elastomer stack can be left on the shim or removed and used separately. Note that many small stacks ( $<10$ layers) may be combined to form larger stacks ( $>100$ layers) as a way of reducing overall manufacturing time.

In summary, the fabrication method described above requires only basic components such as a DC motor, wheel and an airbrush system. The wheel spraying concept has the inherent advantage of giving a consistent layer thickness without requiring a change in direction of the motor or control over its velocity profile. The set-up has been demonstrated to produce individual polymer layers less than $100 \mu \mathrm{m}$ thick. 


\section{Fabrication parameters}

The key parameters considered in the proposed fabrication process are summarised as follows:

\section{Deposition distance}

This is the distance from the airbrush tip to the surface of shim. If this distance is too small, the force of the spray flow will give rise to a distorted and inhomogeneous layer thickness. Too far a distance may result in patchy and inconsistent layers. The deposition distance also affects the maximum width of the layers being produced and therefore the shape of the actuator. In general, the combination of the deposition distance, airbrush system pressure and the spray mixture composition (explained below) will determine the thickness of the layers produced.

\section{Airbrush system pressure}

This is the pressure of the airflow supplied to the airbrush system via a compressor or compressed air canister. Too high a pressure will increase the amount of material wasted during spraying. The pressure also affects how long spraying of a single layer may take, and therefore influence the overall fabrication time. A pressure of 2.5bar was found suitable for the work presented here.

\section{Airbrush nozzle size}

For variable nozzles it is important to keep the nozzle opening constant if a constant layer thickness from one batch to the next is desired. A wider nozzle opening will increase the spray flow rate and sprayed area and hence reduce the time taken to spray a layer. However, this may also increase material wastage. Conversely, a narrower opening will increase spray time but reduce product waste.

\section{Silicone type}

Suitable dielectric materials, such as Nusil CF19-2186, Dow Corning HSIII and BJB TC-5005, are now widely available. Care should be taken with fast curing silicones to ensure curing does not occur while the mixture is still in the spray container as it may block the spray nozzle and affect the consistency of the sprayed elastomer layer. Silicones with a low uncured viscosity and large curing times ( $>24$ hours) may be unsuitable with this fabrication technique as the centrifugal and gravity forces may cause sprayed layers to run prior to curing. Thermally accelerated silicones also exist for which the curing time decreases with increasing temperature. The silicone used in this work is Dow Corning Silastic 3481 condensation cure elastomer and can be used with various curing agents to adjust the speed of curing.

\section{Spray mixture composition}

This is the proportion of silicone to solvent in the spray mixture. A higher proportion of solvent must be used when a large volume of silicone is being spayed or if the silicone has a high uncured viscosity. Evaporation times vary 
between solvents types and may need to be taken into account if layer spraying is to be done over a long period of time as the solvent concentration in the spray container will be decreasing over time. A silicone-solvent ratio of 5:1 by weight was found to be adequate for the work done here.

\section{Electrodes}

Electrodes can be applied in a variety of methods using a variety of materials. Depending on the material being used, electrodes can be airbrushed, hand brushed or smeared onto the silicone surface. Suitable electrode materials for this fabrication process are graphite powder, conductive carbon grease and graphite powder in a silicone matrix. Hand brushed graphite powder was found to yield the best results whilst taking the least amount of time. It is important that the graphite powder is gently brushed on, otherwise the powder tends to clump together increasing the stiffness of the elastomer layer and hence, reduces the performance of the actuator. Care should be taken when using carbon grease electrodes as accumulation of the grease can occur when applying the electrode too close towards the edges of the electrode mask (Figure 6).

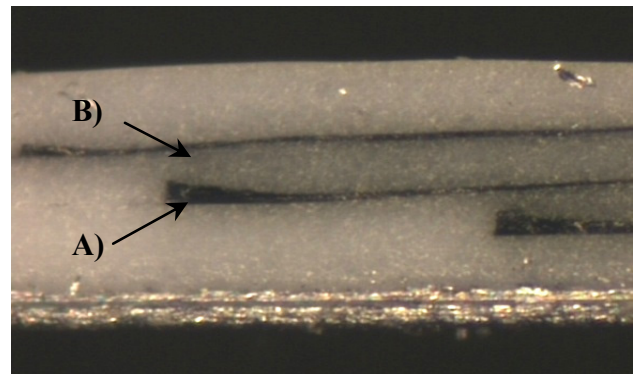

Figure 6: Effects of accumulation of carbon grease electrodes (cross section through width of actuator). A) accumulation of grease, B) region of weakness.

Electrical connection of electrodes in a multilayer configuration can be done in a variety of ways. In this fabrication method, part of the electrode offset region is left exposed and subsequent layers are connected in parallel by applying the electrodes directly on top of each other in the offset region (depicted in Figure 5). Connecting the electrodes in this way allows for flexibility in the design and configuration of the multilayer films when forming functional actuators. Aluminium foil or some other conductive material in flexible form can then be used to connect the exposed regions to the voltage supply.

\section{Environmental conditions}

It is important to manufacture in clean room environment as small particles of dust can adversely affect quality of layers and layer bonding. Figure 7 shows the effect of a dust particle. The figure shows how a particle causes the layers to warp and distort around it. This would have the effect of producing a region of reduced thickness and hence a point of weakness by electric breakdown. 


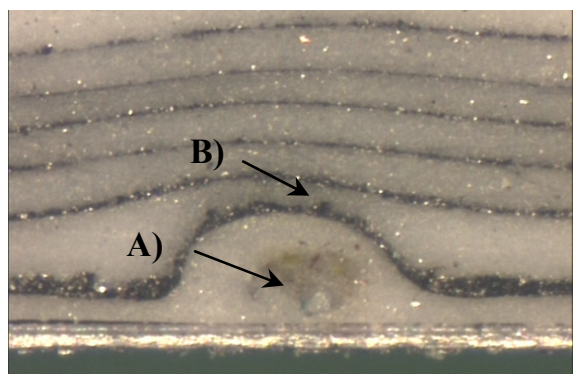

Figure 7: Effect of imperfection on multilayer actuator (cross section through length) A) dust particle B) point of weakness.

\section{Actuator case study}

Figure 8 shows the actuator that has been used as a case study for assessing the validity of the fabrication system developed in this work. The actuator is a 'bender' actuator (multimorph) where bending is induced by constraining the planar deformation of a multilayer stack during activation to produce bending. The planar constraint is provided by the shim substrate used in the fabrication procedure described above.

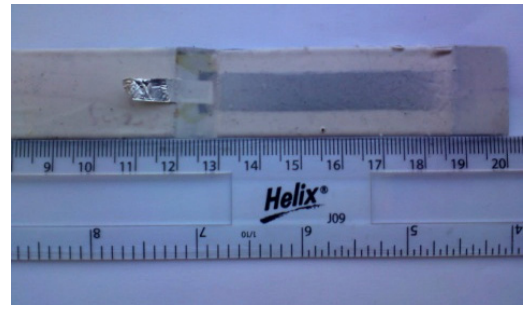

Figure 8: DE multilayer bending actuator made using developed fabrication set-up.

The actuator has 6 layers and each layer is approximately $100 \mu \mathrm{m}$ thick and the active electrode region is $50 \mathrm{~mm}$ long and $10 \mathrm{~mm}$ wide. The total actuator width and free length is $20 \mathrm{~mm}$ and $80 \mathrm{~mm}$ respectively. Hand brushed graphite powder was used for the electrodes which are electrically connected in parallel, as shown schematically in Figure 5 above. The shim forms one terminal and the aluminium foil forms another. DEA driving signals were generated using a National Instruments NI-PCI 6229 DAQ module. The signals were then amplified using a HA-151A potentiostat (Hokuto Denko, Japan) and stepped up to high voltages using an EMCO F121 converter (EMCO High Voltage Corporation), which is capable of supplying up to $12 \mathrm{kV}$. The output voltage and current from the potentiostat were measured using the DAQ module. The tip displacements were measured using a Keyence LK-G152 laser displacement 


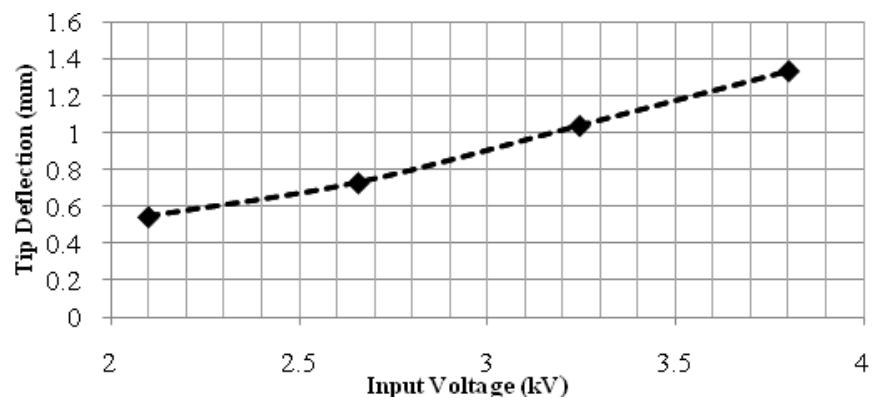

Figure 9: Actuator tip deflection against input voltage for a 6 layer DE bender actuator.

sensor positioned 70mm from the actuator's fixed end. Figure 9 shows the results of tip deflection against input voltage for the 6 layer actuator at four different input voltages.

From [12], the tip displacement of a piezoelectric unimorph actuator is linearly proportional to the actuation stress, which is also proportional to the input voltage, assuming a constant elastic modulus. The DEA bender shares a similar actuation principle with the piezoelectric bender, and hence the tip deflection, in this case, is proportional to the square of the input voltage, according to eqn (1). Figure 9 shows an approximately squared relationship between bender tip deflection and input voltage, hence validating the quality of the proposed fabrication method.

Bender actuators also have the advantage of being well suited for resonant operation, which can improve actuator efficiency and maximise actuator output for a given input voltage. Figure 10 shows the response of the 6 layer actuator to a $5 \mathrm{~Hz}$ square wave input signal of $2.1 \mathrm{kV}$ amplitude and $66.7 \%$ duty cycle. The actuator stroke amplitude reaches a value of $5.8 \mathrm{~mm}$, which is 10 times greater than the static tip deflection for the same input voltage at $2.1 \mathrm{kV}$.

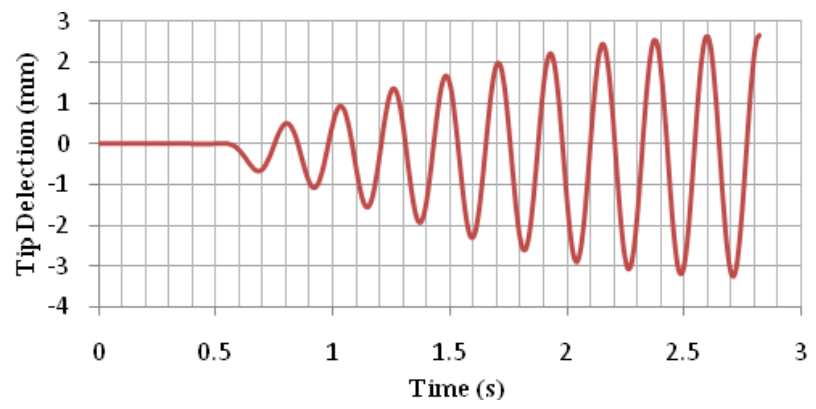

Figure 10: Resonant driving of a 6 layer DE bender actuator. 
Resonating DE bending actuators have potential for use in a variety of bioinspired applications, such as wing flapping in micro air vehicles (MAV) [6]. The flapping-wing MAV design developed at the U.S Naval Postgraduate School (NPS) by Jones and Platzer [13] could also benefit from the incorporation of resonating DEA benders. Their MAV design arose from the numerical and empirical research into oscillating airfoils. The MAV possesses a pair of plunging airfoils that flap together in counter-phase, so that they reproduce the aerodynamic phenomenon known as the 'ground effect' that is exploited in nature by many avian species [14]. The flapping wings are configured to produce thrust, with a static airfoil attached in front of them to produce lift. Resonating benders could improve efficiency and provide improved flight control by using two actuators at either end of each foil connected by a flexible membrane and actuated with different input voltages to create asymmetric aerodynamic forces (Figure 11). This would eliminate the need for a rudder and may improve flight mobility in confined spaces.

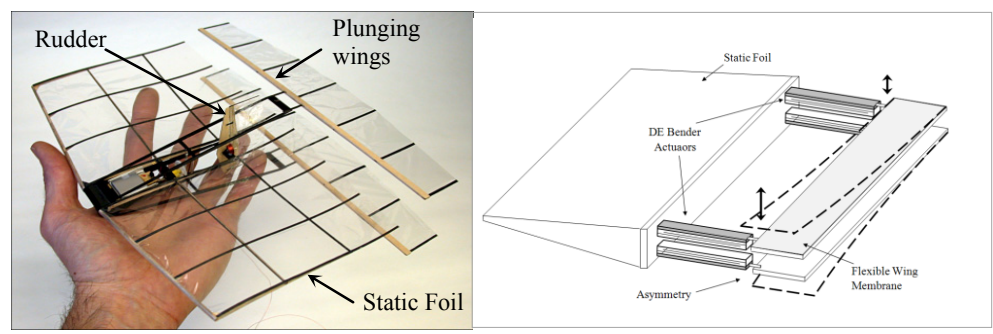

Figure 11: Left: Rudder controlled propulsion system for NPS flapping-wing MAV (Image from [13]). Right: Proposed design for DE driven rudderless propulsion system.

\section{Conclusions}

This paper has presented a novel design of a fabrication set-up for making dielectric elastomer actuator (DEA) EAP actuators that is flexible, relatively simple and inexpensive, and well suited to low-volume production and actuator prototyping. The set-up has been demonstrated to produce individual polymer layers less than $100 \mu \mathrm{m}$ thick. The key fabrication parameters have been outlined and the quality of the method assessed in a case study using a 6 layer bender actuator. Resonant driving of the actuator has also been demonstrated with results showing over 10 times greater stroke amplitude than the static activation case for the same input voltage. A potential application in the realm of bioinspired robotics for such actuators have also been presented.

\section{Acknowledgements}

The authors would like to thank Dr. Jonathan Rossiter for his valuable advice and input and also the Bristol Robotics Laboratory for the use of its facilities. 


\section{References}

[1] Pelrine, R., Kornbluh, R. and Joseph, J. Electrostriction of polymer dielectrics with compliant electrodes as a means of actuation. Sensors and Actuators A: Physical, 64, pp. 77-85, 1998.

[2] Conn, A.T., Burgess, S.C., Ling C.S. and Vaidyanathan, R. The design optimisation of an insect-inspired micro air vehicle. International Journal of Design and Nature and Ecodynamics, 3, pp.12-27, 2008

[3] Bar-Cohen, Y., (ed.). Electroactive polymer (EAP) actuators as artificial muscles - reality, potential and challenges. Second Edition. SPIE Press, Bellingham, WA, U.S.A, 2004.

[4] Pei Q., Pelrine R., Stanford S., Kornbluh R., Rosenthal M. Electroelastomer rolls and their application for biomimetic walking robots. Syn. Met., 135/136, pp. 129-131, 2003.

[5] Carpi, F. and D. D. Rossi. Biomimetic dielectric elastomer actuators. Biomedical Robotics and Biomechatronics, pp. 1073-1078, 2006.

[6] S.C. Burgess, C.S. Ling, A. Conn, S. Araromi, J. Wang and R. Vaidyanathan. Development of a novel Electro Active Polymer (EAP) actuator for driving the wings of flapping micro air vehicle. Computer Aided Optimum Design in Engineering XI. ed. S. Hernandez, C.A. Brebbia, Southampton, WIT Press, pp. 207-217, 2009.

[7] G. Kovacs, L. Düring, S. Michel, G. Terrasi, Stacked dielectric elastomer actuator for tensile force transmission, Sensors and Actuators: A. Physical, 155, pp. 299-307, 2009.

[8] Ha S. M., Yuan ', Pei Q., Pelrine R. and Stanford S. Interpenetrating networks of elastomers exhibiting 300\% electrically-induced area strain. Smart Mater. Struct., 16, pp. S280 - S287, 2007.

[9] P. Lotz, M. Matysek, P. Lechner, M. Hamann, H.F. Schlaak. Dielectric Elastomer Actuators using improved Thin Film Processing and nanosized Particles. Proc. SPIE, 6927, 692723, 2008.

[10] Schlaak, H. F.; Jungmann, M. Matysek, P. Lotz Novel Multilayer Electrostatic Solid State Actuators with Elastic Dielectric, Proc SPIE 5759, pp. 121-133, 2005.

[11] Polypower 2010, www.polypower.com.

[12] Q.M Wang, L. E. Cross, Performance Analysis of Piezoelectric Cantilever Bending Actuators. Ferroelectrics 215, pp. 187-213, 1998.

[13] Jones, K.D. and Platzer, M.F. Bio-inspired design of flapping wing micro air vehicles - an engineer's perspective. 44th Aerospace Sciences Meeting and Exhibit, Reno, 2006.

[14] Jones, K.D., Castro, B.M., Mahmoud, O. and Platzer, M.F. A numerical and experimental investigation of flapping-wing propulsion in ground effect. AAIA 40th Aerospace Sciences Meeting and Exhibit, AIAA Paper No. 2002-0866, Reno, 2002. 